\title{
Rat Substrains Differ in the Magnitude of Spontaneous Locomotor Recovery and in the Development of Mechanical Hypersensitivity after Experimental Spinal Cord Injury
}

\author{
Jacob Kjell, Katalin Sandor, ${ }^{2}$ Anna Josephson,, Camilla I Svensson, ${ }^{2}$ and Mathew B. Abrams ${ }^{1}$
}

\begin{abstract}
A number of different rodent experimental models of spinal cord injury have been used in an attempt to model the pathophysiology of human spinal cord injury. As a result, interlaboratory comparisons of the outcome measures can be difficult. Further complicating interexperiment comparisons is the fact that the rodent response to different experimental models is strain-dependent. Moreover, the literature is abundant with examples in which the same injury model and strain result in divergent functional outcomes. The objective of this research was to determine whether substrain differences influence functional outcome in experimental spinal cord injury. We induced mild contusion spinal cord injuries in three substrains of Sprague-Dawley rats purchased from three different European breeders (Scanbur, Charles River, and Harlan) and evaluated the impact of injury on spontaneous locomotor function, hypersensitivity to mechanical stimulation, and bladder function. We found that Harlan rats regained significantly more hindlimb function than Charles River and Scanbur rats. We also observed substrain differences in the recovery of the ability to empty the bladder and development of hypersensitivity to mechanical stimulation. The Harlan substrain did not show any signs of hypersensitivity in contrast to the Scanbur and Charles River substrains, which both showed transient reduction in paw withdrawal thresholds. Lastly, we found histological differences possibly explaining the observed behavioral differences. We conclude that in spite of being the same strain, there might be genetic differences that can influence outcome measures in experimental studies of spinal cord injury of Sprague-Dawley rats from different vendors.
\end{abstract}

Key words: locomotor function; sensory function; spinal cord injury; strain; substrain

\section{Introduction}

$\mathbf{T}$ RaUmatic INJURY TO THE SPINAL CORD often results in motor, sensory, and sexual dysfunctions, as well as loss of bladder control, hypo- and hypersensitivity, and pain. ${ }^{1}$ Today, there are no treatments that restore impaired function. ${ }^{2}$ Experimentally, researchers use rodent models of spinal cord injury to model the pathophysiological changes found in humans to evaluate the efficacy of experimental treatments. Efforts have been made to standardize experimental injury models such as compression, contusion, and transection models. ${ }^{3-5}$ Noteworthy, different rat strains give different outcomes with the same experimental injury model, which complicates experimental comparisons. ${ }^{6}$ The cause of this has been attributed to genetic differences in the molecular response elicited by the injury. This genetic rationale, however, fails to explain rat substrain differences observed in the literature. ${ }^{7-9}$

Substrain differences in experimental nervous system models in rats have been reported for neuropathic pain behavior, startle reflex, and spinal noradrenergic neuron projections. ${ }^{10-12}$ Nothing, however, has been reported on rat substrain differences in outcome after experimental spinal cord injury. Thus, the aim of this study was to determine whether substrains differ in spontaneous locomotor and sensory recovery after contusion spinal cord injury. For this purpose, we assessed locomotor recovery, hypersensitivity to mechanical stimulation, and bladder recovery in three different substrains of Sprague-Dawley rats.

\section{Methods \\ Spinal cord injury}

The experiment was performed according to the Helsinki declaration and the ethical approval issued by the Northern Stockholm Animal Ethical Committee. Female Sprague-Dawley rats (225$275 \mathrm{~g}, n=30$ ) were divided into three groups based on substrain/ vendor (Scanbur [Germany], Harlan [Netherlands], and Charles River [Germany]) and subjected to mild contusion spinal cord injury. Weights at time of injury for Harlan, Scanbur, and Charles River rats were $240 \pm 2 \mathrm{~g}, 242 \pm 4 \mathrm{~g}$, and $268 \pm 2 \mathrm{~g}$, respectively (supplementary Fig. 1; see online supplementary material at ftp.liebertpub.com). Briefly, the rats were anesthetized using $2 \%$

Departments of ${ }^{1}$ Neuroscience and ${ }^{2}$ Physiology and Pharmacology, Karolinska Institutet, Stockholm, Sweden. 
isoflurane, and the dorsal surface of the spinal cord was exposed through laminectomy of thoracic vertebras T10 and the caudal half of T9. The spinal cord injury was induced by dropping a $10 \mathrm{~g}$ weight from $12.5 \mathrm{~mm}$ above the dorsal surface of the spinal cord using the Impactor (Keck Center for Neurosciences). Rats from the three substrains were alternately injured, and the computerized system of the Keck impactor ensured that the severity of the injury was standardized. A consistent SCI impact force was ensured with measurements of height, time $(\mathrm{Ct})$, and impact velocity (Vi). Rats with errors exceeding $2 \%$ of expected values were excluded. In addition, Harlan rats $(n=8)$ were subjected to a moderate injury ( $25 \mathrm{~mm}$ weight drop) as described above, but with another surgeon.

Analgesic treatment (bruprenophin $0.015 \mathrm{mg} / \mathrm{kg}$, Temelgesic, i.p. ) was administered before surgery and once a day for the following 3 days. Further postoperative care consisted of administering prophylactic antibiotics $(0.6 \mathrm{mg} / \mathrm{kg}$ trimethoprim, Borgal, Hoechst, AG) for 7 days and manual emptying of the bladder twice daily until the animal regained bladder function. Animals had access to food and water ad libitum and were housed three per cage. The temperature was maintained at $24-26^{\circ}$ with $12 \mathrm{~h}$ light and dark cycles.

\section{Assessment}

Open field locomotion test. Hindlimb locomotion and function were assessed in an open field using the Basso, Beattie, Bresnahan (BBB) locomotor rating scale and the BBB subscore. ${ }^{13,14}$ Briefly, two experimenters blinded to group identity assessed hindlimb locomotor function weekly for 8 weeks.

Quantitative locomotion test. Noldus Catwalk System (Noldus IT, The Netherlands) was used for automated assessment of locomotion and stepping parameters, such as the regularity index (a measure of limb coordination), base of support (width in between paws), stance time (time paw touches walkway in seconds), and swing time (time, in seconds, paw is not touching walkway), and stride length (length in between placement of a paw in $\mathrm{cm}$ ). Each animal had to perform three complete runs (continuous locomotion), which were averaged into a single score. Catwalk measurements were done at weeks 2,5 , and 8 post-injury, and the data were normalized to pre-surgical catwalk scores performed 1 week before injury.
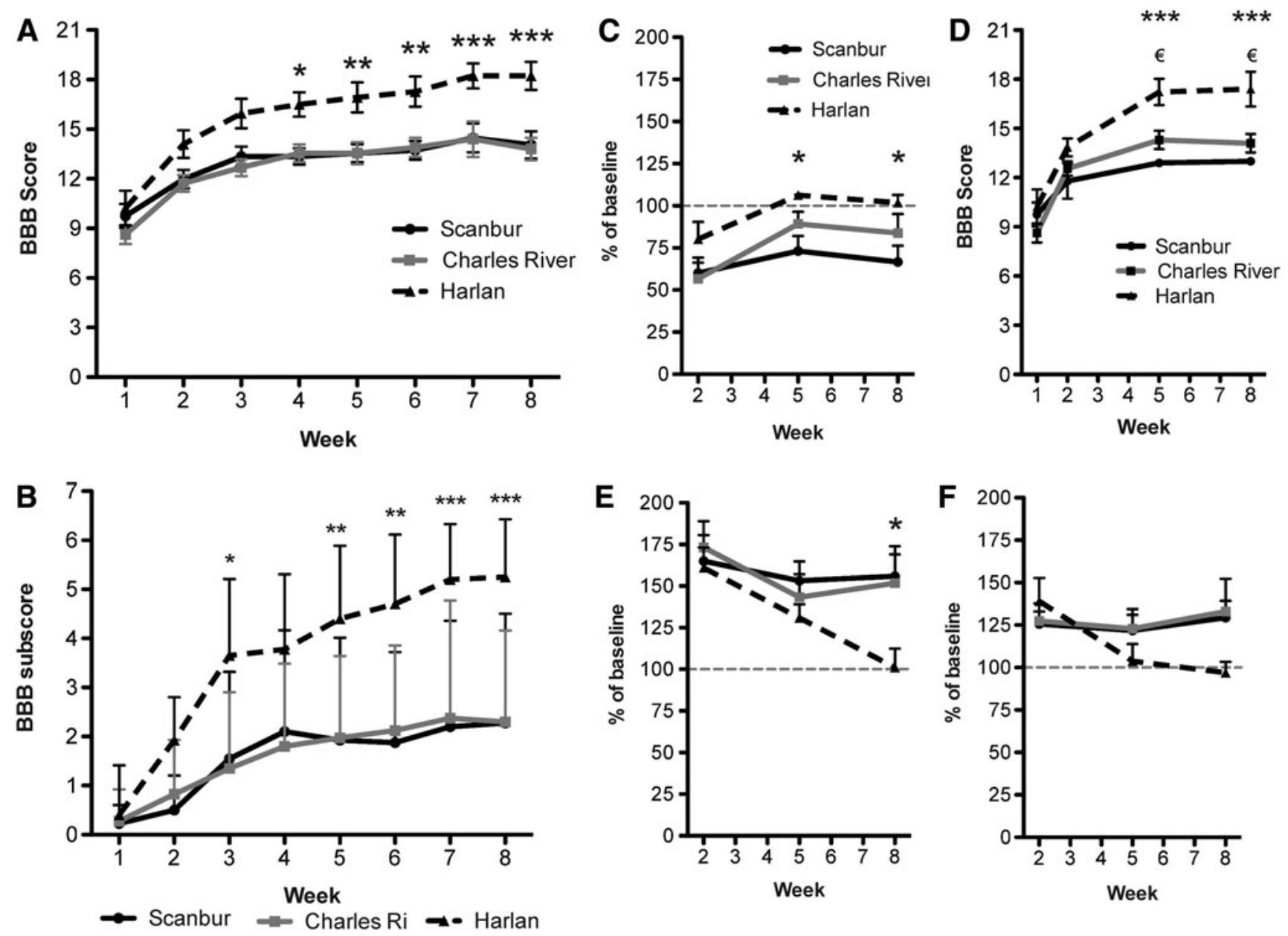

FIG. 1. Substrains differ in spontaneous locomotor recovery. (A) The substrains' locomotor recovery was assessed in an open field and scored with the Basso, Beattie, Bresnahan (BBB) score and (B) BBB subscore. (C) Automated measurement of limb coordination, called the regularity index, measured by the Noldus Catwalk. Harlan versus Charles River, not significant; Harlan versus Scanbur, $p<0.05(*)$. (D) Regularity index integrated BBB score. The automated coordination measurements, called the regularity index, were exchanged for the observed coordination used in the BBB score. Harlan versus Charles River, $p<0.05$; ( $€$ ), Harlan versus Scanbur, $p<0.001$ (***). (E) Catwalk measurements of hindlimb base of support. (F) Catwalk measurements of hindlimb swing time. Data presented as the mean \pm standard error of the mean. $* p<0.05, * * p<0.01, * * * p<0.001$. Subscore presented as the mean \pm standard deviation. $* p<0.05, * * p<0.01, * * * p<0.001$. 
Semi-quantitative locomotion test. At 2, 5, and 8 weeks post-injury, the regularity index score obtained with the Catwalk was integrated into the $\mathrm{BBB}$ locomotor rating scale to obtain a quantitative measure of coordination, instead of the observer dependent measure typical of the BBB locomotor rating scale. ${ }^{15}$

Mechanical hypersensitivity. Rats were habituated to the testing environment twice on separate days. Mechanical hypersensitivity was assessed three times before and once weekly after the injury. Rats were placed in individual Plexiglas enclosures on top of a wire mesh surface and allowed to acclimatize for 30 minutes before testing. Withdrawal thresholds were assessed by using calibrated von Frey filaments (Stoelting, Wood Dale, IL) with approximately logarithmically incremental force of $0.4,1,2,4,6,8$, 10 , and $15 \mathrm{~g}$. A cutoff of $15 \mathrm{~g}$ was applied to avoid tissue damage; thus, suprathreshold responses were not assessed. Each filament was pressed perpendicularly against the center of the hindpaw and held on for approximately $3 \mathrm{sec}$. A positive response was recorded if the paw was withdrawn. The filaments were used according to the "up-down" method and the 50\% probability of withdrawal threshold was calculated as described elsewhere and expressed as percent change from the baseline values. ${ }^{16,17}$ The withdrawal threshold was calculated for both hindpaws and averaged.

Bladder function. After injury, the bladder has to be emptied manually until the animals regain ability to empty their own bladders. We measured the residual urine volume in every animal at 1 day post-injury and then once per week for 4 weeks.

\section{Spinal cord preparation}

At 56 days after injury, under deep anesthesia (pentobarbital, Nembutal, $40 \mathrm{mg} / \mathrm{kg}$, i.p) animals were transcardially flushed with $75 \mathrm{~mL}$ calcium free Tyrode solution containing $0.1 \mathrm{~mL}$ of heparin (5000 IE/mL). Spinal cords were subsequently hydro-extruded and put in fixative (4\% paraformaldehyde in $0.1 \mathrm{M}$ phosphate buffered solution $[\mathrm{PBS}]$ ) for $2 \mathrm{~h}$. Fixed cords were transferred to $10 \%$ sucrose solution, which was exchanged daily for 5 days. The spinal cords were divided into $7 \mathrm{~mm}$ segments spanning $14 \mathrm{~mm}$ caudal and rostral to the injury site. The spinal segments were embedded in OCT media and frozen on dry ice. Cryostat cut sections $(20 \mu \mathrm{m})$ were thaw-mounted onto gelatin-coated slides.

Immunohistochemistry and histology. Antibodies for SMI312 (Covance), GFAP (Dako), and ED1 (Serotec) were used to evaluate axonal loss, astrocyte reactivity, and activated macrophage density, respectively. Primary antibody was applied to cryostat-sectioned spinal cord sections and incubated for $24 \mathrm{~h}$ at $4^{\circ} \mathrm{C}$. Primary antibodies were visualized using secondary antibodies (DyLight ${ }^{\circledR} 488$ and 555, Jackson Laboratories) after a $1.5 \mathrm{~h}$ incubation at room temperature. Antibodies were diluted in $0.3 \%$ Triton $^{\mathrm{TM}}-\mathrm{X}$ in 0.1M PBS. Slides were coated (ProLong ${ }^{\circledR}$ gold antifade reagent) and were visualized using $10 \times$ and $5 \times$ magnification under epifluorescent microscopy (Nikon Eclipse TE3000). Luxol fast blue staining was used to evaluate white matter sparing. When stained, sections were dried and mounted with Entellan ${ }^{\circledR}$. The sections of the injury site were visualized with epifluorescence microscopy (Nikon Eclipse1000) and photographed (Spot camera, Spot Advance software, Tekno optik) at $5 \times($ scale bar $=500 \mu \mathrm{m})$.

Fiji image software (fiji.sc) was used to quantify the area of white matter within the injury site and the area of null neurofilament (SMI-312) immunoreactivity 1-2 mm below the injury. It was also used to quantify glial fibrillary acidic protein and ED1 immunoreactivity as well as cavity perimeter within the injury. In all quantifications, three sections per animal from the investigated area were quantified and averaged, and thus represented the measure for one animal. All animal measures were then used for data graphs and statistical analysis.

\section{Statistics}

Tissue staining and tissue area measurements were analyzed using one-way analysis of variance (ANOVA) followed by the Bonferroni post-hoc test. For the analysis of BBB score, Catwalk data, residual urine, and mechanical hypersensitivity, two-way ANOVA followed by the Bonferroni post-hoc test was used. BBB subscore was analyzed using the nonparametric Mann-Whitney $U$ test. Weights are displayed as mean values with standard error.

\section{Results}

\section{Substrains differ in spontaneous locomotor recovery}

Assessment of the hindlimb function using the BBB scale revealed that Harlan rats recovered more hindlimb function compared with both Scanbur and Charles River rats. Harlan rats had a significantly higher $\mathrm{BBB}$ score compared with the other two substrains from week 4 peaking at a score of $18.2 \pm 0.85$ compared with $14.1 \pm 0.82$ and $13.8 \pm 0.68$ for Scanbur and Charles River rats, respectively, at week 8 (Fig. 1A). The BBB subscore showed a similar pattern with Harlan rats having higher scores than both Scanbur and Charles River rats. At week 8, Harlan rats had a significantly higher subscore of $5.3 \pm 1.18$, while Scanbur rats had a BBB subscore of $2.3 \pm 2.23$ and Charles River rats had a score of $2.3 \pm 1.86$ (Fig. 1B).

There was a significant difference in the regularity index between the Harlan and Scanbur rats at week 8, measuring $101.8 \pm 4.6 \%$ compared with $66.6 \pm 9.7 \%$ (Fig. 1C), while Charles River rats had a regularity index of $83.8 \pm 11.3 \%$.

While coordination is assessed through observations using the $\mathrm{BBB}$ score, the regularity index can serve as an objective measurement of limb coordination. Replacing the observed coordination with the automatically recorded regularity index values leads to a modified BBB score that also shows significantly higher scores for the Harlan rats compared with both the Scanbur and Charles

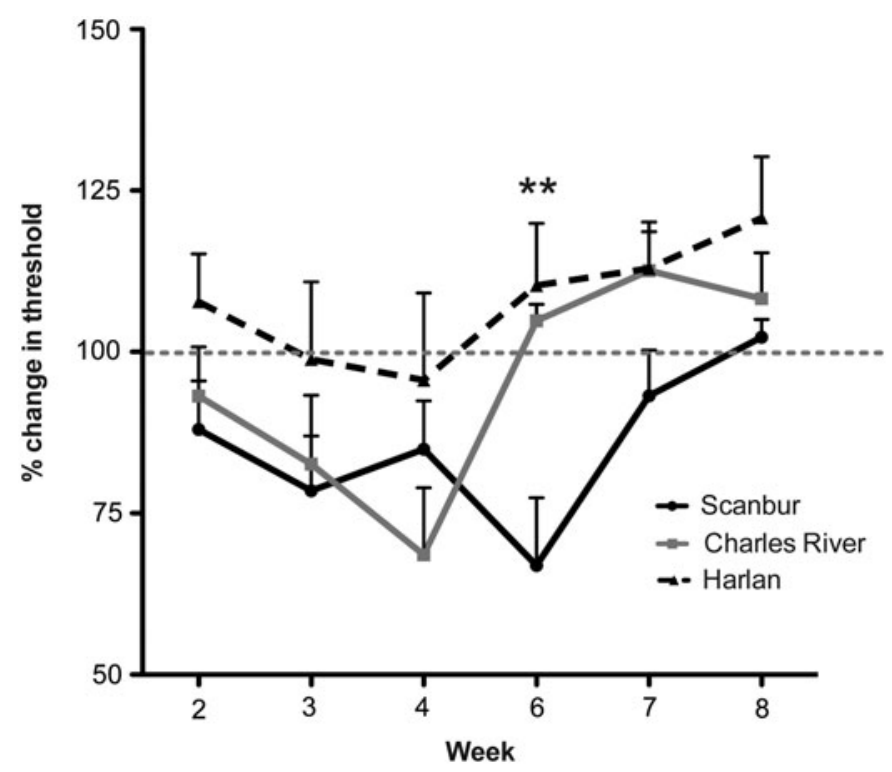

FIG. 2. Substrain differences in development of hypersensitivity. Von Frey filaments were used to detect mechanical hypo- or hypersensitivity of the hindlimbs. Data presented as the mean \pm standard error of the mean. ${ }^{* *} p<0.01$ 


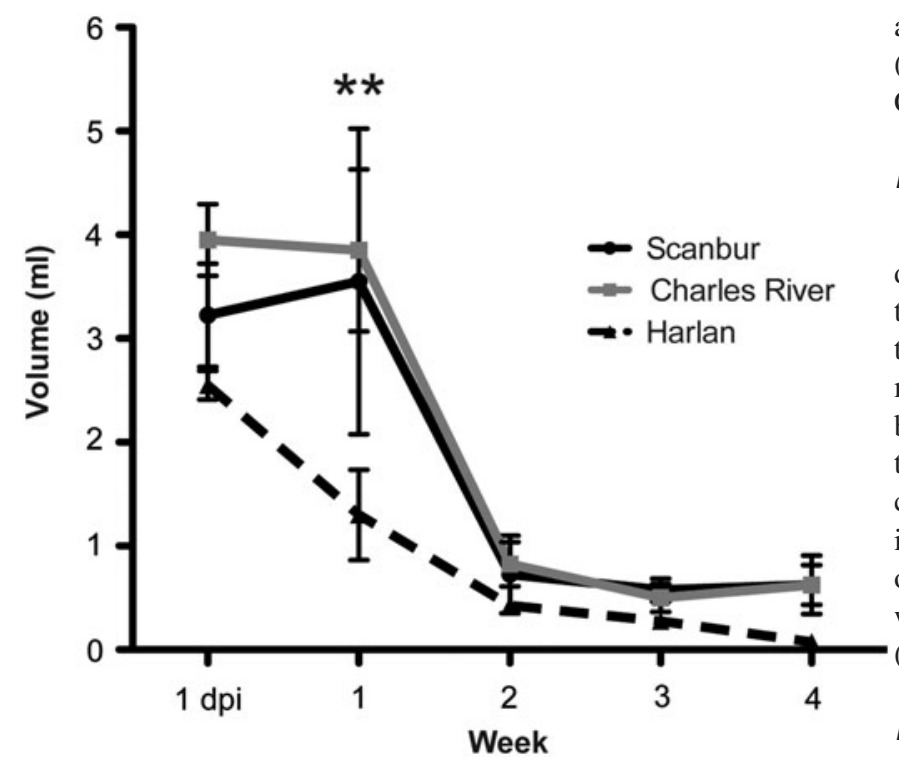

FIG. 3. Substrains differ in recovery of bladder function. Residual urine was measured until 4 weeks after injury when all animals had regained the ability to empty the bladder. Data presented as the mean \pm standard error of the mean. $* * p<0.01$

River rats at weeks 5 and 8. At week 5, scores were 17.2 \pm 0.80 , $12.9 \pm 0.23$ and $14.3 \pm 0.56$, respectively. At week 8, scores were $17.4 \pm 1.05,13.0 \pm 0.21$, and 14.1 \pm 0.57 , respectively (Fig. 1D).

By the end of the 8-week-long experimental period, Harlan rats had normalized stance width $(96.9 \pm 6.5 \%)$, while Scanbur and Charles River rats generally maintained a widened stance to $129.4 \pm 9.9 \%$ and $133.0 \pm 19.3 \%$ compared with baseline values, respectively (Fig. 1E). Increased hindlimb swing time was observed in all three substrains after the injury. This measure had returned to baseline values by week 5 post-injury in Harlan rats (101.1 $\pm 11.2 \%)$, while it remained elevated in the other two subtrains $-155.9 \pm 18.0$ and $151.8 \pm 17.2 \%$ in Scanbur and Charles River rats, respectively (Fig. 1F). There were no baseline differences in any of the analyzed Catwalk measurements (not shown).

\section{Development of mechanical hypersensitivity}

Mechanical hypersensitivity did not develop in Harlan rats during the 8-week period of observation. Mechanical hypersensitivity in the Scanbur substrain developed around week 3 and returned to baseline threshold values by week 8 . Scanbur reached maximum hypersensitivity by week $6(66.9 \pm 10.5 \%$ compared with baseline), which was significantly different compared with both of the other two substrains (Fig. 2). Charles River rats showed similar changes in the withdrawal threshold during the first 3 weeks postinjury; however, the peak hypersensitivity in this substrain was observed at week 4 (68.6 $\pm 10.4 \%$ compared with baseline), and the withdrawal threshold was reversed to baseline values by week 6 (Fig. 2).

\section{Recovery of bladder function}

We measured residual urine 1 day post-injury and then once a week for 4 weeks. Harlan rats had a faster recovery of bladder function compared with both Scanbur and Charles River rats, which showed similar recovery patterns. At 1 week post-injury, Harlan rats had significantly less residual urine, $1.3 \mathrm{~mL}$, compared with $3.6 \mathrm{~mL}$ and $3.9 \mathrm{~mL}$ for Scanbur and Charles River rats, respectively (Fig. 3.). As expected from the mild injury, ability to empty the bladder was regained in all substrains by 3 weeks post-injury.

\section{Histopathological changes in the spinal cord after mild contusion injury}

We measured the area with total or severe loss of neurofilament below the injury site as a measure of loss of descending pathways and measured the area of spared white matter at the injury site. The loss of neurofilaments below the injury site, determined by using SMI-312 (pan-axonal marker), was found to be more prominent in
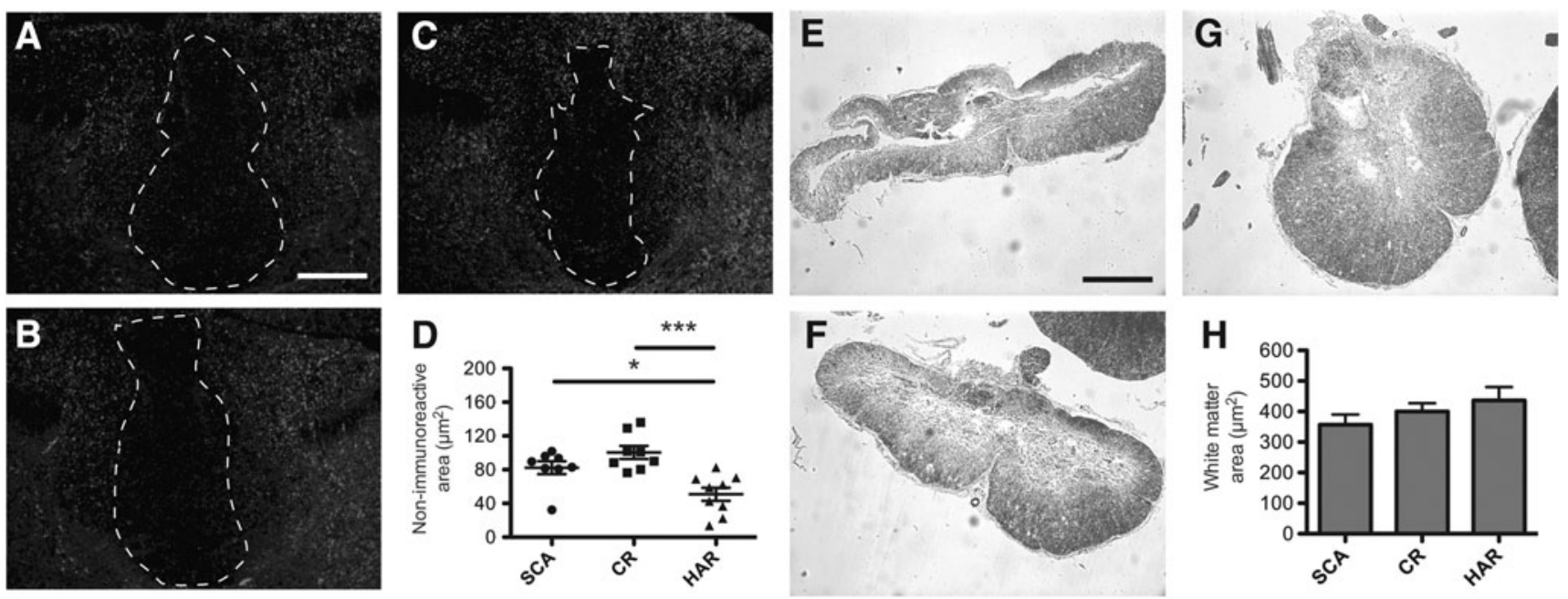

FIG. 4. Greater loss of axons and white matter in the Scanbur (SCA) and Charles River (CR) substrains compared with the Harlan (HAR) substrain. (A) Antibody SMI-312 was used to visualize axonal sparing 1-2 mm below the injury site. Representative images of the spinal cord of the Scanbur substrain, (B) Charles River substrain, and (C) Harlan substrain. (D) Quantification of SMI-312 negative area 1-2 $\mathrm{mm}$ below the injury site. (E) Luxol fast blue staining was used to detect spared myelin at the injury site. Representative images from Scanbur substrain, $(\mathbf{F})$ Charles River substrain, and $(\mathbf{G})$ Harlan substrain. (H) Quantification of myelin at the injury site. Scale bars: $\mathrm{SMI}-312=50 \mu \mathrm{m}, \mathrm{LFB}=250 \mu \mathrm{m}$. Data presented as the mean \pm standard error of the mean. $* p<0.05, * * p<0.01, * * * p<0.001$. 

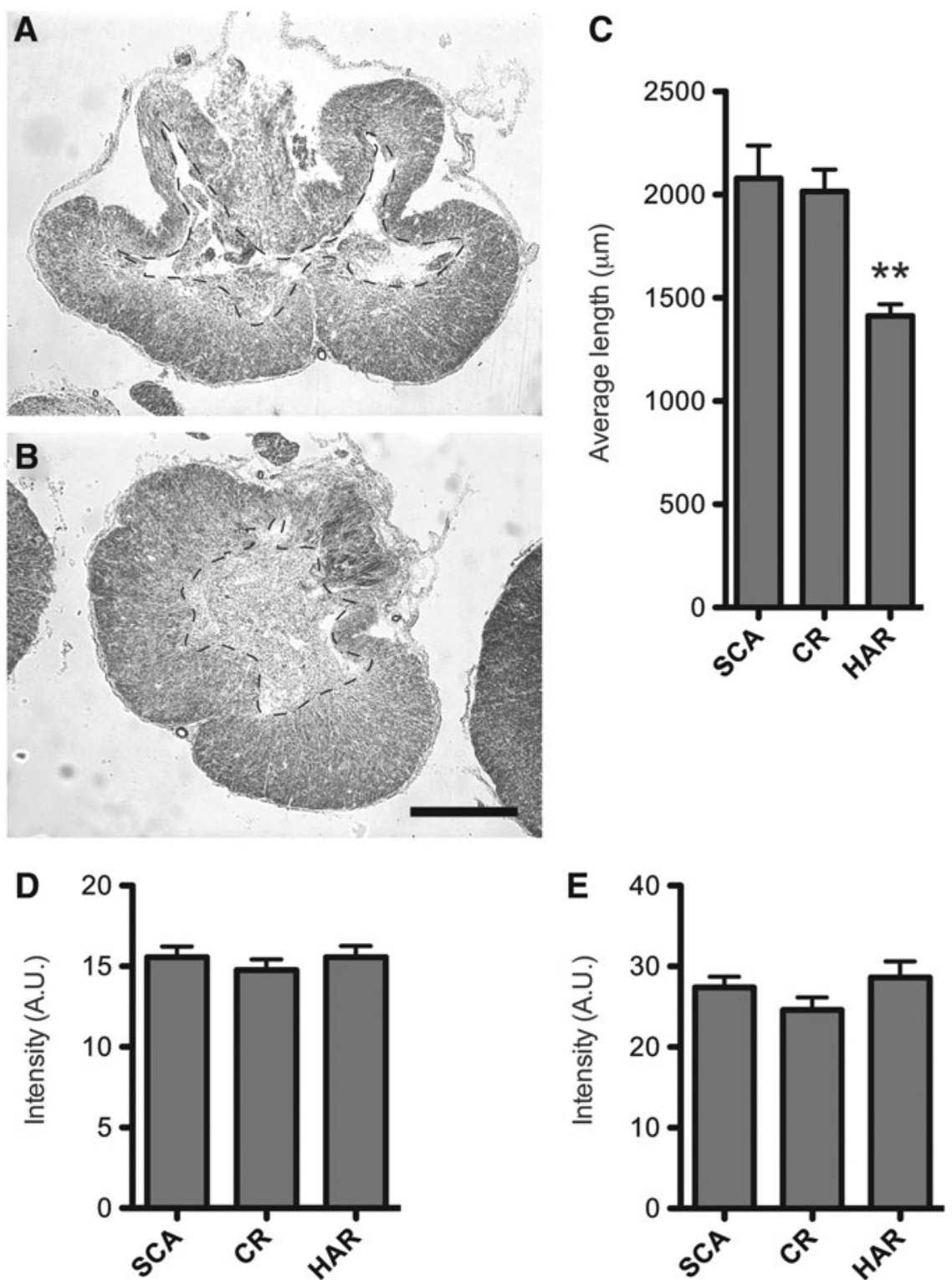

FIG. 5. Substrains differ in tissue morphology, not inflammation. Representative image of the injury site morphology from: (A) Scanbur (SCA) substrain and (B) Harlan (HAR) substrain. (C) Quantification of cavity perimeter of the injury site. (D). Quantification of ED1 immunoreactivity 8 weeks post-injury at the injury site. (E) Quantification of glial fibrillary acidic protein (GFAP) immunoreactivity 8 weeks post-injury at the injury site. Scale bar $=250 \mu \mathrm{m}$. Data presented as the mean \pm standard error of the mean. $* * p<0.01$. $\mathrm{CR}=$ Charles River

Scanbur and Charles River rats, averaging $82 \mu \mathrm{m}$ and $100 \mu \mathrm{m}$ compared with $51 \mu \mathrm{m}$ for Harlan rats (Fig. $4 \mathrm{~A}-\mathrm{D}$ ). At the injury site we found there to be a trend of more white matter spared in favor of Harlan rats (Fig. 4E-H).

We observed differences in degeneration of tissue and cavity formation at the injury site (Fig. 5 A,B). Measuring the perimeter of the cavities of the injury sites, Scanbur and Charles River rats had a significantly longer perimeter, $2.1 \mathrm{~mm}$ and $2 \mathrm{~mm}$, compared with $1.4 \mathrm{~mm}$ for Harlan rats (Fig. 5C).

We investigated the injury-induced immune response to test for macrophage/microglia activation and astrocyte reactivity, by measuring ED1 and GFAP immunoreactivity, respectively, at the injury site. We found that neither the density of astrocytes or macrophages/microglia differed among the substrains at 8 weeks post-injury (Fig. 5D-E).

\section{Discussion}

We investigated whether substrains of the commonly used Sprague-Dawley rat differ in the magnitude of spontaneous structural and functional recovery from mild spinal contusion injury and found that the substrain purchased from the vendor Harlan recovered significantly better compared with the rats purchased from Charles River and Scanbur. Harlan rats recovered better hindlimb locomotor function and had a reduced period of bladder dysfunction than the two other Sprague-Dawley substrains. Further, transient mechanical hypersensitivity was detected in both Scanbur and Charles River rats, while no change in withdrawal thresholds was found in the Harlan substrain.

The improved spontaneous functional recovery might in part be from the enhanced tissue preservation and smaller spinal cysts that 
were observed in the Harlan rats compared with the other two substrains. The distinct pattern of tissue preservation observed in the Harlan rats may contain parts of the rubrospinal tract, which can compensate for some of the motor functions of the corticospinal tract and is reportedly a better indicator of functional recovery than the general sparing of white matter. ${ }^{18-21}$ Interestingly, it has been reported that the anatomical location of spinal neuron projections can vary between rat substrains, so it is plausible that the improved functional outcome observed in the Harlan rats is because of a somewhat different anatomic location of key axon pathways in the spinal cord. ${ }^{11}$

The strain differences previously found in rats was attributed to anatomical differences and genetic differences in the molecular response elicited by the injury. ${ }^{6,22,23}$ Other factors affecting the injury response could include the size and shape of the spinal canal and physical properties of the dura mater. ${ }^{23,24}$

In addition, increased tissue preservation and function has been positively correlated with reduced inflammation. Under the current experimental conditions, we did not observe any significant differences in magnitude of chronic inflammation between the substrains. This observation does not rule out the possibility of strain differences with respect to inflammatory/immune response during the acute phase of injury.

Our results indicate that the differences in the magnitude of spontaneous locomotor recovery between substrains of SpragueDawley rats can be as large as those observed when comparing different strains of rats. Harlan rats recovered significantly more spontaneous locomotor function compared with the other two strains. We observed a similar effect when injuring the SpragueDawley substrains with a moderate contusion injury. Interexperimental differences in spontaneous locomotor recovery within the same rat strain have typically been attributed to the use of different injury types, surgical skill, or the skill level of those performing the functional assessments. While this may be the case in some instances, our results indicate that there may also be true differences specific to the particular substrain used.

We found that the three European Sprague-Dawley rat substrains were not universally suited for all types of functional assessments (i.e., locomotor, mechanical hypersensitivity, and bladder testing). Minor motor functional deficits developed in Harlan rats after a mild contusion injury and hypersensitivity to mechanical stimuli did not develop; however, even after moderate contusion injury (supplementary Fig. 2; see online supplementary material at ftp.liebertpub.com), Harlan rats typically regain the ability to walk, making them a suitable choice for the testing of treatment-induced mechanical hypersensitivity with von Frey filaments. Moderate motor functional deficits and hypersensitivity after a mild contusion injury developed in Scanbur rats. After moderate contusion injury, Scanbur rats typically do not regain the ability to support weight, so they are not a good choice for hypersensitivity testing. ${ }^{25}$ Similar moderate motor functional deficits as the Scanbur rats and hypersensitivity after a mild contusion injury developed in Charles River Sprague-Dawley rats. We found that after a moderate injury, a transient flaccid paralysis of the bladder develops in many Charles River rats, and they show a high mortality rate. Collectively, our results highlight the importance of substrain selection in experimental spinal cord injury research.

\section{Conclusion}

We have presented evidence in support of rat substrain differences with respect to spontaneous recovery after experimental contusion spinal cord injury. Our results also indicate the importance of selecting the most appropriate substrain whose behavior will be best assessed by the experimental design, and highlight the role that substrain can play in comparing results from different experiments and groups. Continued work into the mechanisms underlying the substrain differences is warranted. Moreover, investigations of the same substrains tested here using other spinal cord injury models, such as transection or compression, are also warranted.

\section{Acknowledgment}

We thank professor Lars Olson for constructive review of the manuscript. Supported by Vinnova, Wings for Life, the Swedish Research Council, The Karolinska DPA award, Wenner-Gren Foundation (KS), Swedish Research Council (CIS), International Association for the Study of Pain (CIS) and Karolinska institutet.

\section{Author Disclosure Statement}

No competing financial interests exist.

\section{References}

1. Wrigley, P.J., Press, S.R., Gustin, S.M., Macefield, V.G., Gandevia, S.C., Cousins, M.J., Middleton, J.W., Henderson, L.A., and Siddall, P.J. (2009). Neuropathic pain and primary somatosensory cortex reorganization following spinal cord injury. Pain 141, 52-59.

2. Sayer, F.T., Kronvall, E., and Nilsson, O.G. (2006). Methylprednisolone treatment in acute spinal cord injury: the myth challenged through a structured analysis of published literature. Spine J. 6, 335-343.

3. Metz, G.A., Curt, A., van de Meent, H., Klusman, I., Schwab, M.E., and Dietz, V. (2000). Validation of the weight-drop contusion model in rats: a comparative study of human spinal cord injury. J. Neurotrauma 17, 1-17.

4. Scheff, S.W., Rabchevsky, A.G., Fugaccia, I., Main, J.A., and Lumpp, J.E. Jr. (2003). Experimental modeling of spinal cord injury: characterization of a force-defined injury device. J. Neurotrauma 20, 179-193.

5. Basso, D.M., Beattie, M.S., and Bresnahan, J.C. (1996). Graded histological and locomotor outcomes after spinal cord contusion using the NYU weight-drop device versus transection. Exp. Neurol. 139, 244-256.

6. Mills, C.D., Hains, B.C., Johnson, K.M., and Hulsebosch, C.E. (2001). Strain and model differences in behavioral outcomes after spinal cord injury in rat. J. Neurotrauma 18, 743-756.

7. Liu, X.Z., Xu, X.M., Hu, R., Du, C., Zhang, S.X., McDonald, J.W., Dong, H.X., Wu, Y.J., Fan, G.S., Jacquin, M.F., Hsu, C.Y., and Choi, D.W. (1997). Neuronal and glial apoptosis after traumatic spinal cord injury. J. Neurosci. 17, 5395-5406.

8. Ozawa, H., Keane, R.W., Marcillo, A.E., Diaz, P.H., and Dietrich, W.D. (2002). Therapeutic strategies targeting caspase inhibition following spinal cord injury in rats. Exp. Neurol. 177, 306-313.

9. Yu, C.G., Jimenez, O., Marcillo, A.E., Weider, B., Bangerter, K., Dietrich, W.D., Castro, S., and Yezierski, R.P. (2000). Beneficial effects of modest systemic hypothermia on locomotor function and histopathological damage following contusion-induced spinal cord injury in rats. J. Neurosurg. 93, Suppl 1, 85-93.

10. Swerdlow, N.R., Martinez, Z.A., Hanlon, F.M., Platten, A., Farid, M. Auerbach, P., Braff, D.L., and Geyer, M.A. (2000). Toward understanding the biology of a complex phenotype: rat strain and substrain differences in the sensorimotor gating-disruptive effects of dopamine agonists. J. Neurosci. 20, 4325-4336.

11. Clark, F.M., and Proudfit, H.K. (1992). Anatomical evidence for genetic differences in the innervation of the rat spinal cord by noradrenergic locus coeruleus neurons. Brain Res. 591, 44-53.

12. Yoon, Y.W., Lee, D.H., Lee, B.H., Chung, K., and Chung, J.M. (1999). Different strains and substrains of rats show different levels of neuropathic pain behaviors. Exp. Brain. Res. 129, 167-171.

13. Basso, D.M., Beattie, M.S., and Bresnahan, J.C. (1995). A sensitive and reliable locomotor rating scale for open field testing in rats. J. Neurotrauma 12, 1-21. 
14. Lankhorst, A.J., Verzijl, M.R., and Hamers, F.P. (1999). Experimental spinal cord contusion injury: Comparison of different outcome parameters. Neurosci. Res. Com. 24, 135-148.

15. Koopmans, G., Deumens, R., Honig, W.M., Hamers, F.P., Steinbusch, H.W., and Joosten, E.A. (2005). The assessment of locomotor function in spinal cord injured rats: the importance of objective analysis of coordination. J. Neurotrauma 22, 214-225.

16. Dixon, W.J. (1980). Efficient analysis of experimental observations. Annu. Rev. Pharmacol. Toxicol. 20, 441-462.

17. Chaplan, S.R., Bach, F.W., Pogrel, J.W., Chung, J.M., and Yaksh, T.L. (1994). Quantitative assessment of tactile allodynia in the rat paw. J. Neurosci. Methods 53, 55-63.

18. Raineteau, O., and Schwab, M.E. (2001). Plasticity of motor systems after incomplete spinal cord injury. Nat. Rev. Neurosci. 2, 263-273.

19. Brown, L.T. (1974). Rubrospinal projections in the rat. J. Comp. Neurol. 154, 169-187.

20. Metz, G.A., Dietz, V., Schwab, M.E., and van de Meent, H. (1998). The effects of unilateral pyramidal tract section on hindlimb motor performance in the rat. Behav. Brain Res. 96, 37-46.

21. Schucht, P., Raineteau, O., Schwab, M.E., and Fouad, K. (2002). Anatomical correlates of locomotor recovery following dorsal and ventral lesions of the rat spinal cord. Exp. Neurol. 176, 143-153.

22. Popovich, P.G., Wei, P., and Stokes, B.T. (1997). Cellular inflammatory response after spinal cord injury in Sprague-Dawley and Lewis rats. J. Comp. Neurol. 377, 443-464.
23. Dimar, J.R., II, Glassman, S.D., Raque, G.H., Zhang, Y.P., and Shields, C.B. (1999). The influence of spinal canal narrowing and timing of decompression on neurologic recovery after spinal cord contusion in a rat model. Spine (Phila Pa 1976) 24, 16231633 .

24. Maikos, J.T., Elias, R.A., and Shreiber, D.I. (2008). Mechanical properties of dura mater from the rat brain and spinal cord. J. Neurotrauma $25,38-51$.

25. Abrams, M.B., Nilsson, I., Lewandowski, S.A., Kjell, J., Codeluppi, S., Olson, L., and Eriksson, U. (2012). Imatinib enhances functional outcome after spinal cord injury. PLoS ONE 7, e38760.

Address correspondence to: Jacob Kjell, MSc

Department of Neuroscience Karolinska Institutet Retzius väg 8, B2:4 17177 Stockholm Sweden

E-mail: jacob.kjell@ki.se 